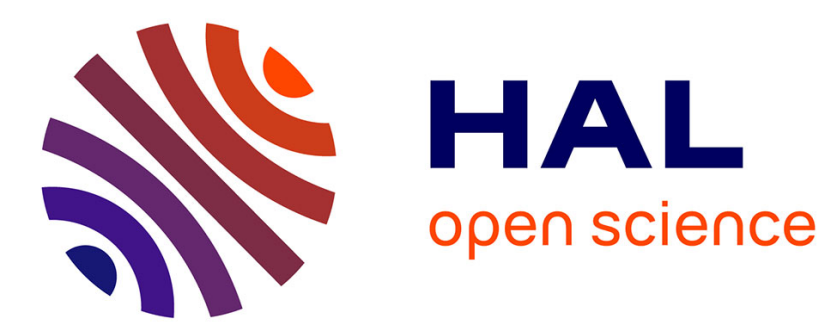

\title{
Competing Technologies, Technological Monopolies and the Rate of Convergence to a Stable Market Structure
}

Andrea Bassanini, Giovanni Dosi

\section{To cite this version:}

Andrea Bassanini, Giovanni Dosi. Competing Technologies, Technological Monopolies and the Rate of Convergence to a Stable Market Structure. 1999. halshs-00185579

\section{HAL Id: halshs-00185579 \\ https://shs.hal.science/halshs-00185579}

Preprint submitted on 6 Nov 2007

HAL is a multi-disciplinary open access archive for the deposit and dissemination of scientific research documents, whether they are published or not. The documents may come from teaching and research institutions in France or abroad, or from public or private research centers.
L'archive ouverte pluridisciplinaire HAL, est destinée au dépôt et à la diffusion de documents scientifiques de niveau recherche, publiés ou non, émanant des établissements d'enseignement et de recherche français ou étrangers, des laboratoires publics ou privés. 


\section{Competing Technologies, Technological Monopolies,}

and the Rate of Convergence to a Stable Market Structure*

\section{Andrea P. Bassanini ${ }^{\dagger}$}

\section{Giovanni Dosi $i^{\ddagger}$}

November 29, 1999

*The views expressed here cannot be ascribed to the OECD Secretariat or its Member Countries. We are indebted to Yuri Kaniovski for very helpful suggestions. We thank also Brian Arthur, Robin Cowan, Klaus Desmet, Judith Gebauer, Michael Horvath, Andrea Prat, Aldo Rustichini, Valter Sorana, and participants to the 3rd workshop on Economics with Heterogeneous Interacting Agents, Ancona, Italy, May 1998, and to the Conference on Economic Models of Evolutionary Dynamics and Interacting Agents, Trieste, Italy, September 1998, for their comments. Financial support from International Institute for Applied Systems Analysis (IIASA), Banca Nazionale del Lavoro (BNL), Italian Research Council (CNR), and Italian Ministry of University and Scientific Research (MURST) is gratefully acknowledged. All errors are ours.

†OECD, Paris, France. E-mail: andrea.bassanini@oecd.org.

`Sant’Anna School for Advanced Studies, Pisa, Italy. E-mail: g.dosi@sssup.it. 
Empirically the diffusion of competing technologies most often displays either "lock-in" to a quasi-monopoly or apparent turbulence but rarely stable market-sharing. In contrast with widespread views, we show that, first, unbounded increasing returns are neither necessary nor sufficient to lead to technological monopolies. Rather, asymptotic patterns depend on the relative impact of increasing returns and the degree of adopters heterogeneity. Second, the unlikely empirical occurrence of stable market-sharing is interpreted in terms of different rates of convergence. We show that convergence to market-sharing is slower than to monopoly; thus, in the former case, the environment often changes before the market-share trajectory becomes stable.

JEL Classification: O33, L11, C60

Keywords: Competing Technologies, Product Selection, Unbounded Returns, Network Externalities, Heterogeneity, Technological Monopolies. 


\section{Introduction}

In this paper we address the dynamics of diffusion of different technologies competing for the same market niche.

The stylized fact at the origin of this work is the observation that a stable empirical pattern of market sharing between competing technologies with no overwhelming dominant position rarely occurs in markets with positive feedbacks ${ }^{1}$. For example, even in the case of operating systems, which is often quoted as a case of market sharing, Apple MacIntosh has never held a market share larger than 1/5 (a partial exception being the submarket of personal computer for educational institutions). This fact has also triggered suspicion of market inefficiencies: Technological monopolies may prevail even when the survival of more than one technology may be socially optimal [Katz and Shapiro (1986), David (1992)]. Think for example to the competition between Java-based architectures and ActiveX architectures for web-based applets: Given that with any of the two paradigms the standard tasks that can be performed are different, the general impression of experts is that society would benefit from the survival of both.

In turn, from the point of view of interpretation of the processes of diffusion of new products and technologies, it is acknowledged that, in many modern markets, they are characterised by increasing returns to adoption or positive feedbacks. This has partly to do with supply-side causes: the cumulation of knowledge and skills through the expansion of markets and production usually reduce the hedonic price of both production and consumption goods, thus increasing the net benefit for the user of a particular technology. Boeing 727, for example, which has been on the jet aircraft market for years, has undergone constant modification of the design and improvement in structural soundness, wing design, payload capacity and engine efficiency as it accumulates airline adoption and hours of flight [Rosenberg (1982), Arthur (1989)]. Similar observations can be made for many helicopter designs [Saviotti and Trickett (1992)] as well as for electric power plants designs [Cowan (1990), Islas (1997)].

Supply-side causes of this type have received some attention in the economic literature for quite a while. However in the last fifteen years a great deal of attention has been devoted also to demand-side positive feedbacks,

${ }^{1}$ See e.g. the empirical literature on dominant designs [for a recent survey, cf. Tushman and Murmann (1998)]. 
so-called network esternalities or (more neutrally) network effects [Katz and Shapiro (1994), Liebowitz and Margolis (1994)]. For example, telecommunication devices and networks (for instance fax machines), as a first approximation, tend not to provide any utility per se but only as a function of the number of adopters of compatible technologies with whom the communication is possible [Rohlfs (1974), Oren and Smith (1981), Economides (1996)]. The benefits accruing to a user of a particular hardware system depend on the availability of software whose quantity and variety may depend on the size of the market if there are increasing returns in software production. This is the case of VCRs, microprocessors, hi-fi devices and in general systems made of complementary products which need not be consumed in fixed proportions [Cusumano et al. (1992), Church and Gandal (1993), Katz and Shapiro $(1985,1994)]$. A similar story can be told for the provision of postpurchase service for durable goods. In automobile markets, for example, the diffusion of foreign models has often been slow because of consumers' perception of a thinner and less experienced network of repair services [Katz and Shapiro (1985)]. Standardization implies also saving out of the cost of investment in complementary capital if returns from investment are not completely appropriable: In software adoption firms can draw from a large pool of experienced users if they adopt software belonging to a widespread standard, thus de facto sharing the cost of training [Farrell and Saloner (1986), Brynjolfsson and Kemerer (1996)]. Moreover product information may be more easily available for more popular brands or, finally, there may be conformity or psychological bandwagon effects [Katz and Shapiro (1985), Banerjee (1992), Arthur and Lane (1993), Bernheim (1994), Brock and Durlauf (1995)].

Katz and Shapiro (1994) in their review of the literature on systems competition and dynamics of adoption under increasing returns distinguish between technology adoption decision and product selection decision.

The former refers to the choice of a potential user to place a demand in a particular market. Relevant questions in this case are the conditions for an actual market of positive size, the notional features of a "socially optimal" market size and the conditions allowing penetration of a new (more advanced) technology into the market of an already established one [Rohlfs (1974), Oren and Smith (1981), Farrell and Saloner (1985,1986), Katz and Shapiro (1992)]. For example purchasing or not a fax or substituting a compact disc player for an analogical record player are technology adoption decisions.

Conversely product selection refers to the choice between different technological solutions which perform (approximately) the same function and are 
therefore close substitutes. Relevant questions here are whether the market enhances variety or standardization, whether the emerging market structure is normatively desirable and what is the role of history in the selection of market structure [Arthur $(1983,1989)$, Katz and Shapiro $(1985,1986)$, David (1985), Church and Gandal (1993), Dosi et al. (1994)]. Choosing between VHS or Beta in the VCR market or between Word or Wordperfect in the wordprocessors market are typical examples of product selection decisions.

This work is concerned with the dynamics of product selection. To explain the stylized fact recalled above we analyze properties of a fairly general and nowadays rather standard class of models of competing technologies, originally suggested by Arthur (1983) and Arthur et al. (1983) and further explored and popularized by Arthur (1989), Cowan (1991), and Dosi et al. (1994), among others. This class of models will be presented in details in section 2.

Despite mixed results of some pioneering work on the dynamics of markets with network effects [e.g. Katz and Shapiro (1986)], unbounded increasing returns are commonly called for as an explanation of the emergence of technological monopolies. Usually the argument is based on the results of the model set forth by Arthur (1989). For instance Robin Cowan summarizes it in the following way:

"If technologies operate under dynamic increasing returns (often thought of in terms of learning-by-doing or learning-by-using), then early use of one technology can create a snowballing effect by which that technology quickly becomes preferred to others and comes to dominate the market.

"Following Arthur, consider a market in which two types of consumers adopt technology sequentially. As a result of dynamic increasing returns arising from learning-by-using, the payoff to adopting a technology is an increasing function of the number of times it has been adopted in the past. Important with regard to which technology is chosen next is how many times each of the technologies has been chosen in the past. Arthur shows that if the order of adopters is random (that is, the type of the next adopter is not predictable) then with certainty one technology will claim the entire market" [Cowan (1990) p. 543, italics added].

It will be shown in the following that this statement does not always hold. 
Unbounded increasing returns to adoption are neither necessary nor sufficient to lead to the emergence of technological monopolies. As proved in the next section, strictly speaking, Arthur's result applies only when returns are linearly increasing and the degree of heterogeneity of agents is, in a sense, small. Moreover it cannot be easily generalized further: Some meaningful counterexamples will be provided. More generally the emergence of technological monopolies depends on the nature of increasing returns with respect to the degree of heterogeneity of the population. Relatedly, given a sufficiently high heterogeneity amongst economic agents, limit market sharing may occur even in the presence of unbounded increasing returns. The bearing of our analysis, in terms of the interpretation of the empirical evidence, stems from the results presented in section three: In essence, we suggest that the observation of the widespread emergence of monopolies is intimately related to the properties of different rates of convergence (to monopoly and to market sharing respectively) more than to the properties of limit states as such. It will be shown that a market can approach a monopoly with a higher speed than it approaches any feasible limit market shares where both technologies coexist. Following a line of reasoning put forward by Winter (1986) among others, our argument proceeds by noticing that when convergence is too slow the external environment is likely to change before any sufficiently small neighborhood of the limit can be attained. The result that we obtain, based on some mathematical properties of generalized urn schemes ${ }^{2}$, is general for this class of models. The empirical implication is that among markets with high rate of technological change and increasing returns to adoption, a prevalence of stable monopolies over stable market-sharing should be observed.

The applications of Arthur's result have gone far beyond the dynamics of competing technologies and typically extended to the role of history in selecting the equilibrium in any situation wherein complementarities are relevant. The analysis of industry location patterns is a case to the point [e.g. Arthur (1990), Krugman (1991a, 1991b), Venables (1996)]. As James Rauch puts it:

"In Arthur's model, firms enter the industry in sequence. Each firm chooses a location on the basis of how many firms are there at the

${ }^{2}$ Throughout this paper we label the generalization of Polya urn schemes set forth by Hill et al. (1980) as generalized urn scheme. That generalization is the most popular in economics but obviously it is not the only possible one [see e.g. Walker and Muliere (1997)]. 
time of entry and a random vector that gives the firm's tastes for each possible location. If agglomeration economies are unbounded as the number of firms increases, then as the industry grows large, one location takes all but a finite set of firms with probability one." [Rauch (1993) pp. 843-44, italics added].

The implications of our results extend to this domain of analysis as well.

The remainder of the paper is devided as follows. Section 2 reviews standard models of competing technologies and provides counterexamples to Arthur's main result. Section 3 establishes our main results on rate of convergence to a stable market structure and builds upon that an alternative explanation for observable patterns of dynamics between competing technologies. Section 4 briefly summarizes the results.

\section{Competing Technologies Revisited: Are Unbounded Increasing Returns Sufficient for the Emergence of Technological Monopolies?}

The class of competing technology dynamics models that we consider takes as the only two basic assumptions the fact that adopters enter the market in a sequence which is assumed to be exogenous, and each adopter makes its adoption choice only once. More than one agent can enter the market in each period [e.g. Katz and Shapiro (1986)] but in order to simplify the treatment we abstract from this complication. The simple theoretical tale that underlies these models can be summarized as follows:

Every period a new agent enters the market and chooses the technology which is best suited to its requirements, given its preferences, information structure and the available technologies. Preferences can be heterogeneous and a distribution of preferences in the population is given. Information and preferences determine a vector of payoff functions (whose dimension is equal to the number of available technologies) for every type of agent. Because of positive (negative) feedbacks, such as increasing (decreasing) returns to adoption, these functions depend on the number of previous adoptions. When an agent enters the market it compares the values of these functions (given its preferences, the available information, and previous adoptions) and chooses the technology which yields the maximum perceived payoff. Which "type" of agent enters the market at any given time is a stochastic event 
whose probability depends on the distribution of types (i.e. of preferences) in the population. Because of positive (negative) feedbacks, the probability of adoption of a particular technology is an increasing (decreasing) function of the number of previous adoptions of that technology.

More formally we can write a general reduced form of payoff functions of the following type:

$$
\Pi_{j}^{i}(\vec{n}(t))=h_{i}\left(a_{j}^{i}, \vec{n}(t)\right),
$$

where $j \in D, D$ is the set of possible technologies, $i \in S, S$ is the set of possible types, $\vec{n}(t)$ is a vector denoting number of adoptions for each technology at time $t\left(n^{j}(t)\right.$ is the number of adoptions of technology $j$ at time $\left.t\right)$, $\vec{a}_{i}$ represents the network-independent components of agent $i$ 's preferences ( $a_{i}^{j}$ identifies a baseline payoff for agents of type $i$ from technology $j$ ), and $h_{i}($.$) is an increasing (decreasing) function (that can differ across agents)$ capturing increasing (decreasing) returns to adoption. Information and expectations are incorporated in $h_{i}($.$) . If, at time t$, an agent of type $i$ comes to the market, it compares the payoff functions choosing $A$ if and only if ${ }^{3}$ :

$$
\Pi_{A}^{i}(\vec{n}(t))=\arg \max _{j \in D}\left\{\Pi_{j}^{i}(\vec{n})\right\} .
$$

1 can be seen as describing an equilibrium reaction function. Consequently, strategic behaviors (including sponsoring activities from the suppliers of technologies) are not ruled out by the foregoing formalization.

In the remainder of this paper we assume that the order of agents entering the market is random, hence $i(t)$ can be considered as an iid sequence of random variables whose distribution depends on the distribution of the population of potential adopters. With this assumption, the dynamics of the foregoing model can be seen in terms of generalized urn schemes:

Consider the simplest case where two technologies, say $A$ and $B$, compete for a market. Let us denote $A$ 's market share with $X(t)$. Given the relationships between (a) total number of adoptions of both technologies $n(t)=t-1+n^{A}(0)+n^{B}(0)$, (b) the current market share $X(t)$ of $A$, and (c) number of adoptions of one specific technology, $n^{i}(t), i=A, B$, that is, $n^{A}(t)=n(t) X(t)$, the dynamics of $X(t)$ is given by the recursive identity

$$
X(t+1)=X(t)+\frac{\xi^{t}(X(t))-X(t)}{t+n^{A}(0)+n^{B}(0)} .
$$

\footnotetext{
${ }^{3}$ We assume that, if there is a tie, agents choose technology $A$. Qualitatively, breaking the tie in a different way would not make any difference.
} 
Here $\xi^{t}(x), t \geq 1$ are random variables independent in $t$ such that

$$
\xi^{t}(x)=\left\{\begin{array}{lll}
1 & \text { with probability } & f(t, x) \\
0 & \text { with probability } & 1-f(t, x)
\end{array},\right.
$$

and $\xi^{t}(\cdot)$ is a function of market shares dependent on the feedbacks in adoption. $f(t, x)$ equals the probability that (1) is true when $X(t)=x$ and is sometimes called urn function. Denoting $\xi^{t}(x)-E\left(\xi^{t}(x)\right)=\xi^{t}(x)-f(t, x)$ with $\zeta^{t}(x)$ we have

$$
X(t+1)=X(t)+\frac{[f(t, X(t))-X(t)]+\zeta^{t}(X(t))}{t+n^{A}(0)+n^{B}(0)} .
$$

Provided that there exist a limit urn function $f(\cdot)$ (defined as that function $f($.$) such that f(t,$.$) tends to it as t$ tends to $\infty)$ and the following condition is satisfied

$$
\sum_{t \geq 1} t^{-1} \sup _{x \in[0,1] \cap R(0,1)}|f(t, x)-f(x)|<\infty,
$$

where $R(0,1)$ is the set of rational numbers in $(0,1)$, attainable limit market shares with positive probability can be found by analyzing the properties of the function

$$
g(x)=f(x)-x=\lim _{t \rightarrow \infty} f(t, x)-x .
$$

Particularly, treating $g(x)$ in the same way of the right hand side of an ordinary differential equation, it is possible to show that the process (2) converges almost surely to the set of stable zeroes ${ }^{4}$. The foregoing formal representation is employed for every result of the present paper.

${ }^{4} \mathrm{~A}$ convenient review of analytical results on generalized urn schemes can be found in Dosi et al. (1994). The reader is referred to that for the results that are not proved in this paper. Particularly, $X(\cdot)$ converges almost surely, as $t$ tends to infinity, to the set of appropriately defined zeroes of the function $g(x)=f(x)-x$. However since we are not going to restrict ourselves to the case when $g(\cdot)$ is a continuous function, we need some standard definition concerning equations with discontinuous functions. For a function $g(\cdot)$ given on $R(0,1)$ and a point $x \in[0,1]$ set

$$
\begin{aligned}
& \underline{a}(x, g)=\inf _{\left\{y_{k}\right\} \subset R(0,1)} \liminf _{k \rightarrow \infty} g\left(y_{k}\right), \\
& \quad \bar{a}(x, g)=\sup _{\left\{y_{k}\right\} \subset R(0,1)} \operatorname{lim\operatorname {sup}_{k\rightarrow \infty }} g\left(y_{k}\right),
\end{aligned}
$$

where $\left\{y_{k}\right\}$ is an arbitrary sequence converging to $x$. Then the set of zeroes $A(g)$ of $g(\cdot)$ 
In some cases, eq. (1) can be expressed directly in terms of shares rather than total numbers; in this case $f(.,$.$) is independent of t$ and (3) is trivially verified.

The foregoing formal model can be better visualized by looking at some well-known example. Consider for instance the celebrated example of the VCR market. JVC's VHS and Sony's Beta were commercialized approximately at the same time. According to many studies [see Cusumano et al. (1992) and Liebowitz and Margolis (1994)] none of the two standards has ever been perceived as unambiguously better and, despite their incompatibility, their features were more or less the same, due to the common derivation from the U-matic design. For these reasons the relevant decisions were likely to be sequential. First, a consumer chooses whether or not to adopt a VCR - technology adoption decision in Katz and Shapiro's terminology -, then, once the adoption decision has been made, it devotes its mind to choose which type of VCR to purchase - product selection decision - (in general it can be expected that most of the consumers buy one single item and not both). Network effects in this market come mainly from increasing returns in design specialization and production of VCR models (so that historically all firms specialized just in one single standard) on the supply side, and from increasing returns externalities and consequent availability of home

on $[0,1]$ is defined by the following relation

$$
A(g)=\{x \in[0,1]:[\underline{a}(x, g), \bar{a}(x, g)] \ni 0\} .
$$

Note that for a continuous $g(\cdot)$ this definition gives the roots of the equation $g(x)=0$ in the conventional meaning. One particular class of attainable singleton components comprises the downcrossing or stable ones, i.e. the points where $f(x)-x$ changes its sign from plus to minus. More precisely, $\theta \in R(0,1)$ is said to be stable if there exists $\epsilon>0$ such that for every $\delta \in(0, \epsilon)$

$$
\inf _{\delta \leq|x-\theta| \leq \epsilon}[f(x)-x](x-\theta)<0 .
$$

If $\theta \in R(0,1)$ is stable then $X(\cdot)$ converges to $\theta$ with positive probability for some initial combination $\vec{n}(0)$. If in addition to $\left(^{*}\right)$

$$
f(x) \in(0,1) \text { for all } x \in R(0,1),
$$

then it converges with positive probability to $\theta$ for any initial combination $\vec{n}(0)$. Finally if the urn function does not have touchpoints and the set $A(g)$ with $g(x)=f(x)-x$ is composed only of singleton components then almost surely the process converges to the set of stable components. 
video rental services on the demand side [Cusumano et al. (1992)]. Despite technical similarities between the two standards, preferences were strongly heterogeneous, due mainly to a brand-name-loyalty type of consumer behavior, which was exploited (especially by JVC) through Original Equipment Manufacturers (OEM) agreements with firms with well-established market shares in electronic durable goods.

The size of VCR market is sufficiently large (hundreds of millions of sold units) to make it approximable by the abstract concept of an infinite capacity market. Therefore, the asymptotic dynamics of this market can be meaningfully analyzed through the asymptotics of generalized urn schemes.

Many other markets display somewhat similar characteristics (for instance spreadsheets, wordprocessors, computer keyboards, pc-hardwares, automobiles etc...). In particular, in many markets product selection can be assumed to sequentially follow technology adoption decisions ${ }^{5}$. The fact that the decision is sequential suggests that product selection decisions might be dependent on market shares rather than on the absolute size of the network. In this case the urn scheme would be even more simplified, with the urn function independent of $t$.

Arthur $(1983,1989)$ considers a payoff function of the following type:

$$
\Pi_{j}^{i}(\vec{n}(t))=a_{j}^{i}+r\left(n^{j}(t)\right)
$$

where $j=A, B, i \in S, S$ is the set of possible types [in the simplest case, considered also in the foregoing quotation from Cowan (1990), $S=\{1,2\}$ ], and $r$ is an increasing function (common for every agent) capturing increasing returns to adoption. If, at time $t$, an agent of type $i$ comes to the market, it compares the two payoff functions choosing $A$ if and only if:

$$
\Pi_{A}^{i}(\vec{n}(t)) \geq \Pi_{B}^{i}(\vec{n}(t)) .
$$

that is

$$
a_{A}^{i}+r\left(n^{A}(t)\right) \geq a_{B}^{i}+r\left(n^{B}(t)\right) .
$$

Suppose that which type of agent enters the market at time $t$ is the realization of an iid random variable $i(t)$. Thus (2) implies that the agent coming to the market chooses $A$ with probability

$$
\mathcal{P}(A(t))=F_{\theta}\left(r\left(n^{A}(t)-r\left(n^{B}(t)\right)\right),\right.
$$

${ }^{5}$ For instance, in the data set of Computer Intelligence InfoCorp employed by Breuhan (1996), more than $80 \%$ of the firms in the sample report using a single word processing package. 
where $F_{\theta}(\cdot)$ denotes the distribution function of $\theta(t)=a_{B}^{i}(t)-a_{A}^{i}(t)$.

From these considerations Arthur's main theorem was derived:

Theorem 1 (Arthur (1989), theorem 3) If the improvement function $r$ increases at least at rate $\epsilon>0$ as $n^{j}$ increases, the adoption process converges to the dominance of a single technology, with probability one.

The proof of the theorem delivered by Arthur is based on theorem 3.1 of Arthur et al. (1986). In fact it is easy to check that in this case, whatever the distribution of $a_{j}$ is, the limit urn function $f(\cdot)$ is a step function defined in the following way:

$$
f(x)=\left\{\begin{array}{lll}
1 & \text { if } & x>1 / 2 \\
F_{\theta}(0) & \text { if } & x=1 / 2 \\
0 & \text { if } & x<1 / 2
\end{array}\right.
$$

A generalized urn scheme characterized by an urn function such as (5) converges to $\{0,1\}$ with probability $1^{6}$.

However theorem 3.1 of Arthur et al. (1986) is not applicable here because condition (3) does not hold in this case. Actually the urn functions are defined by:

$$
f_{t}(x)=F_{\theta}\left(r\left(x\left(t+n^{w}+n^{b}\right)\right)-r\left((1-x)\left(t+n^{w}+n^{b}\right)\right)\right) .
$$

Moreover for $t>K>0, t$ even, they are such that $f_{t}(0)=0, f_{t}(1 / 2)=$ $F_{\theta}(0), f_{t}(1)=1$ and they are continuous in a left neighborhood (which depends on $t$ ) of $1 / 2$; therefore

$$
\sup _{x \in[0,1] \cap R(0,1)}\left|f_{t}(x)-f(x)\right| \geq \min \left\{F_{\theta}(0), 1-F_{\theta}(0)\right\}
$$

which is constant with respect to $t^{7}$.

Even though Arthur's proof is wrong, the theorem is right and an ad hoc proof can be constructed by showing that $n^{A}(t)-n^{B}(t)$ is a time-homogeneous Markov chain with two absorbing barriers [Bassanini (1997), proposition 2.1, provides a complete proof along these lines]. However this result strictly depends on the fact that the function $r(\cdot)$ is asymptotically linear or more

${ }^{6}$ See note 4 above, or Dosi et al. (1994), theorems 1 and 3.

${ }^{7}$ To be precise Arthur (1989) quotes also Arthur et al. (1983), though there the properties are stated only as yet-to-be-proved good sense conjectures. 
than linear. Arthur's result is not generalizable to any type of unbounded increasing returns. Both in the case of increasing returns that are diminishing at the margin and in the case of heterogeneous increasing returns it is possible to find simple examples where convergence to technological monopolies is not an event with probability 1.

Let us illustrate all this by means of two straightforward counterexamples.

Example 1 Let us assume that increasing returns have the common sense property that the marginal contribution to social benefit of, say, the 100th adopters is larger than that of, say, the 100,000th and that this contribution tends asymptotically to zero; formally this means that $\frac{d}{d n^{j}} f\left(n^{j}\right)>0$, $\frac{d^{2}}{d n^{j 2}} f\left(n^{j}\right)<0$ and $\lim _{n^{j} \rightarrow \infty} \frac{d}{d n^{j}} f\left(n^{j}\right)=0$ [this class of functions has been considered by Katz and Shapiro (1985)].

Focusing on the case set forth by Robin Cowan in the foregoing quotation, let us assume that there are only two types of agents $(i=1,2)$ and two technologies. Recall Arthur's payoff functions (4), $\Pi_{j}^{i}(\vec{n}(t))=a_{j}^{i}+r\left(n^{j}(t)\right)$, and assume that $r(\cdot)=s \log (\cdot)$ is a function (which is common for every agent: $s$ is a constant) that formalizes unbounded increasing returns to adoption. Agent $i$ chooses technology $A$ if and only if $\Pi_{A}^{i}(\vec{n}(t)) \geq \Pi_{B}^{i}(\vec{n}(t))$. By taking the exponential on both sides and rearranging we have:

$$
\frac{X(t)}{1-X(t)} \geq e^{\frac{1}{s}\left(a_{B}^{i}(t)-a_{A}^{i}(t)\right)}
$$

The function of the attributes of agent's type which is on the right hand side can be considered a random variable because, as discussed above, $i(t)$ is a random variable. Moreover they are iid because $i(t)$ is iid. Denoting the random variables on the right hand side with $\varsigma(t)$, from $(6)$ we have that the adoption process can be seen as a generalized urn scheme with urn function given by:

$$
f(x)=F_{\varsigma}(x /(1-x)),
$$

where $F_{\varsigma}(\cdot)$ is the distribution function of $\varsigma(t)$. Because $i(t)$ takes just two values $(1,2)$, also $\varsigma(t)$ takes just two values:

$$
\varsigma(t)=\left\{\begin{array}{ll}
e^{\frac{1}{s}\left(a_{B}^{1}-a_{A}^{1}\right)} & \text { with probability } \alpha \\
e^{\frac{1}{s}\left(a_{B}^{2}-a_{A}^{2}\right)} & \text { with probability } 1-\alpha
\end{array},\right.
$$

where we have assumed without loss of generality that

$$
a_{B}^{1}-a_{A}^{1} \leq a_{B}^{2}-a_{A}^{2} .
$$


Thus $F_{\varsigma}$ is by construction a step function with two steps:

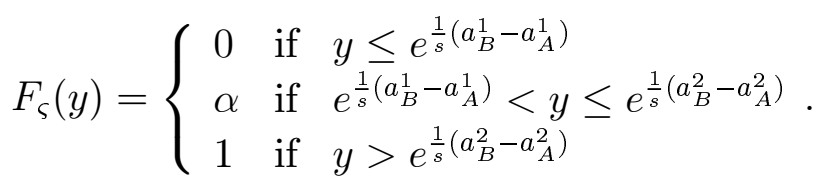

Therefore, taking into account (7), we have that the urn function has two steps and is defined in the following way:

$$
f(x)=\left\{\begin{array}{lll}
0 & \text { if } \quad x \leq \frac{e^{\frac{1}{s}\left(a_{B}^{1}-a_{A}^{1}\right)}}{1+e^{\frac{1}{s}\left(a_{B}^{1}-a_{A}^{1}\right)}} \\
\alpha & \text { if } \quad \frac{e^{\frac{1}{s}\left(a_{B}^{1}-a_{A}^{1}\right)}}{1+e^{\frac{1}{s}\left(a_{B}^{1}-a_{A}^{1}\right)}}<x \leq \frac{e^{\frac{1}{s}\left(a_{B}^{2}-a_{A}^{2}\right)}}{1+e^{\frac{1}{s}\left(a_{B}^{2}-a_{A}^{2}\right)}} . \\
1 \quad \text { if } \quad x>\frac{e^{\frac{1}{s}\left(a_{B}^{2}-a_{A}^{2}\right)}}{1+e^{\frac{1}{s}\left(a_{B}^{2}-a_{A}^{2}\right)}}
\end{array}\right.
$$

If the following condition is satisfied

$$
\frac{e^{\frac{1}{s}\left(a_{B}^{1}-a_{A}^{1}\right)}}{1+e^{\frac{1}{s}\left(a_{B}^{1}-a_{A}^{1}\right)}}<\alpha<\frac{e^{\frac{1}{s}\left(a_{B}^{2}-a_{A}^{2}\right)}}{1+e^{\frac{1}{s}\left(a_{B}^{2}-a_{A}^{2}\right)}},
$$

the urn function has five fixed points, three of which are downcrossing, therefore there is a set of initial conditions (that imply giving both technologies a chance to be chosen "at the beginning of history") for which market sharing is asymptotically attainable with positive probability ${ }^{8}$. The above condition imply that the ratios $\left(e^{a_{A}^{i}}\right)^{1 / r} /\left(e^{a_{B}^{i}}\right)^{1 / r}$ are sufficiently different between the two types. In other words there might be sufficient heterogeneity among agents to counterbalance the effect of increasing returns to adoption ${ }^{9}$.

Example 2 Consider now payoff functions of this type:

$$
\Pi_{j}(\vec{n}(t))=a_{j}+r_{j} n^{j}
$$

where $r_{j}, a_{j}, j=A, B$, are bounded random variables which admit density. Such functions allow agents to be heterogeneous also in terms of the degree of increasing returns which they experience. By applying (1), dividing

${ }^{8}$ See note 4 above, or Dosi et al. (1994), theorem 2.

${ }^{9}$ Cowan and Cowan (1998) acknowledge this role of heterogeneity, although only for models where interactions are local. They suggest that many models from other scientific disciplines can be adapted to show market sharing survival as a result of local interaction effects, and they provide one such example, although restricted to linear returns. 
payoff functions by total number of adoptions, and rearranging we have that $A$ is chosen if and only if:

$$
X(t) \geq \frac{r_{B}}{r_{A}+r_{B}}+\frac{a_{B}-a_{A}}{\left(t+n^{A}(0)+n^{B}(0)\right)\left(r_{A}+r_{B}\right)} .
$$

Denoting the random variables on the right hand side with $\varsigma(t)$, from $(8)$ we have that the adoption process can be seen as a generalized urn scheme with urn function $f(t, x)=F_{\varsigma(t)}(x)$, where $F_{\varsigma(t)}(\cdot)$ is the distribution function of $\varsigma(t)$. Now suppose that $r_{A}$ and $r_{B}$ are highly correlated and both have bimodal distributions very concentrated around the two modes, in such a way that the distribution of $r_{A} / r_{B}$ is also bimodal and very concentrated around the two modes too. Furthermore suppose that the two modes are far away from each other. To fix the ideas say that for a percentage $\alpha$ of the population $r_{A} / r_{B}$ is uniformly distributed on the interval $\left[\frac{1}{1-b}, \frac{1}{1-a}\right]$, while for a percentage $1-\alpha$ of the population $r_{A} / r_{B}$ is uniformly distributed on the interval $\left[\frac{1}{1-d}, \frac{1}{1-c}\right]$, with $0<a<b<c<d$. First, let us consider the case of $a_{j}=0, j=A, B . F_{\varsigma}$ is by construction independent of $t$, implying the following urn function:

$$
f(x)=F_{\varsigma(t)}(x)= \begin{cases}0 & \text { if } y \leq a \\ \alpha \frac{1}{b-a}(y-a) & \text { if } a<y \leq b \\ \alpha & \text { if } b<y \leq c \\ \alpha+(1-\alpha)\left[\frac{1}{d-c}(y-c)\right] & \text { if } c<y \leq d \\ 1 & \text { if } y>d\end{cases}
$$

If $b<\alpha<c$, then there are three stable fixed point of $f(x)$ and, as said above, it can be shown that there is a set of initial conditions (that imply giving both technologies a chance to be chosen "at the beginning of history") for which market sharing is asymptotically attainable with positive probability. If $a_{j} \neq$ 0 but have bounded support and admit density, then condition (3) applies and the same argument holds: In fact, relying on the fact that $r_{j}, a_{j}$ are bounded it is easy to show that $\sup _{x \in[0,1] \cap R(0,1)}|f(t, x)-f(x)|<K / t$, where $K>0$ is a constant. The essential ingredient of this example is that the distribution of $r_{A} / r_{B}$ is bimodal and very concentrated around the two modes. The argument has nothing to do with the particular (and extreme) distributional form assumed here: Following the same constructive procedure adopted here it is easy to build examples with any other distributional form. The only requirement is that the two modes are sufficiently distant. In other words the only requirement is a sufficient degree of heterogeneity in the population 
to counterbalance the pro-standardization effects of increasing returns to adoption

The two examples above show that the degree of increasing returns needs to be compared to the degree of heterogeneity. Unbounded increasing returns that are diminishing fast at the margin are not sufficient to generate asymptotic survival of only one technology, provided that agents are not completely homogeneous [see also Farrell and Saloner $(1985,1986)$ for early models with homogeneous agents that lead to the survival of only one technology]. Even more interesting, when heterogeneity is so wide that there are agent-specific increasing returns, the emergence of technological monopolies is not guaranteed even with returns that are linearly increasing.

To summarize, the foregoing examples show that if preferences are sufficiently heterogeneous and/or increasing returns to adoption are less than asymptotically linear, then Arthur's result cannot be generalized and variety in the asymptotic distribution of technologies can be an outcome with positive probability.

From the point of view of empirical predictions, at first look, the foregoing results might sound, if anything, as a further pessimistic note on "indeterminacy". That is, not only "history matters" in the sense that initial small events might determine which of the notional, technologically attainable, asymptotic states the system might "choose": More troubling, the argument so far suggests that, further, the very distribution of the fine characteristics and preferences of the population of agents might determine the very nature of the attainable asymptotic states themselves. Short of empirically convincing restrictions on the distribution of agents (normally unobservable) characteristics, what we propose is instead an interpretation of the general occurrence of technological monopolies (cum increasing returns of some kind) grounded on the relative speed of convergence to the underlying (but unobservable) limit states.

\section{Rate of Convergence in One-Dimensional Models of Competing Technologies}

In the example of the VCR market, as well as for many other markets, the possibility of predicting limit market shares depends on the feasibility of formalizing the structure of the market in question in terms of a specific urn function. Heterogeneity of preferences, the degrees of increasing returns, the 
type of expectations, price-policies of producers, all affect the functional form of the urn function. As said before, the goal of this paper in general and of this section in particular is to provide some general asymptotic results that can be used as guidance for the interpretation of the empirical evidence on emergence of dominant designs.

Propositions 2, and 4 suffices to the task. Together they imply the relevant statements on the rate of convergence to technological monopoly or to a limit market share where both technologies coexist ${ }^{10}$. Furthermore the analysis that follows applies even in the absence of a clear pattern of increasing returns to adoption. In essence, in the presence of constant returns to adoption, the urn function would be completely constant but the following theorems would still hold.

As above, denote the urn function with $f(\cdot, \cdot)$; the following proposition gives a first result on the rate of convergence to 0 and 1 .

Proposition 1 Let $\epsilon>0$ and $c<1$ be such that eventually

$$
\begin{aligned}
& f(t, x) \leq c x \text { for } x \in(0, \epsilon) \\
& \quad(f(t, x)) \geq 1-c(1-x) \text { for } x \in(1-\epsilon, 1)) .
\end{aligned}
$$

Then for any $\delta \in(0,1-c)$ and $\tau>0$

$$
\begin{aligned}
& \lim _{t \rightarrow \infty} \mathcal{P}\left\{t^{1-c-\delta} X(t)<\tau \mid X(t) \rightarrow 0\right\}=1 \\
& \quad\left(\lim _{t \rightarrow \infty} \mathcal{P}\left\{t^{1-c-\delta}[1-X(t)]<\tau \mid X(t) \rightarrow 1\right\}=1\right),
\end{aligned}
$$

where $X(\cdot)$ stands for the random process given by $(2)$.

The proposition is proved in the appendix.

A similar result can be expressed in terms of variances $\left(L^{2}\right.$-convergence):

Proposition 2 Let $\epsilon>0$ and $c<1$ be such that eventually (9) holds. Then for any $\delta \in(0,1-c)$

$$
\begin{aligned}
& \lim _{t \rightarrow \infty} t^{2(1-c)-\delta} \mathcal{P}\{X(t) \rightarrow 0\} \quad \int_{\{X(t) \rightarrow 0\}} X(t)^{2} d \mathcal{P}=0 \\
& \left(\lim _{t \rightarrow \infty} t^{2(1-c)-\delta} \mathcal{P}\{X(t) \rightarrow 1\} \int_{\{X(t) \rightarrow 1\}}(X(t)-1)^{2} d \mathcal{P}=0\right) .
\end{aligned}
$$

${ }^{10}$ Notice that, provided that inequalities (9) and (10) are eventually satisfied for any $t$, proposition 1, 2 and 4 hold even if the inequality (3) does not hold as may happen when agents are assumed to be forward looking. 
The proposition is proved in the appendix

Notice that proposition 2 states that the rate of convergence of the mean square distance from the limit market share is of the order of $1 / t$ as $t \rightarrow \infty$, conditional to the fact that the process is actually converging to 0 or 1 . Roughly speaking it defines the rate of convergence of mean square errors when the process converges to a technological monopoly. If the set of limit market shares that the process can reach with positive probability contains only these two points, proposition 2 implies a similar statement in terms of the unconditional mean square distance from the limit market share.

One would like to derive a counterpart of proposition 1 and 2 for the case of market sharing, whenever this can be attained with positive probability. For a differentiable $f(\cdot)$ at $0(1)$, (9) holds with $c$ arbitrarily close to $\frac{d}{d x} f(0)\left(\frac{d}{d x} f(1)\right)$. We can easily derive a similar result for a differentiable $f(\cdot)$ independent of $t$ from the following conditional limit theorem for the generalized urn scheme.

Theorem 2 (Arthur et. al. (1987)) Let $\theta \in(0,1)$ be a stable root of $f(x)-x=0$ and $f(\cdot)$ is differentiable at $\theta$ with $\frac{d}{d x} f(\theta)<1 / 2$. Then for every $y \in(-\infty, \infty)$

$$
\lim _{t \rightarrow \infty} \mathcal{P}\left\{\sqrt{t \frac{1-2 \frac{d}{d x} f(\theta)}{2 \theta(1-\theta)}}[X(t)-\theta]<y \mid X(t) \rightarrow \theta\right\}=\Phi(y),
$$

where $\Phi(\cdot)$ stands for the Gaussian distribution function having zero mean and variance 1 .

From this theorem, we can give an even better characterization of the lowest possible convergence rate for a limit market share where both technology coexist that can be attained with positive probability. Indeed, the next proposition follows immediately:

Proposition 3 Let $\theta \in(0,1)$ be a stable root and let

$$
[f(x)-\theta](x-\theta)>k(x-\theta)^{2} \quad \text { for } \quad x \in(\theta-\epsilon, \theta+\epsilon), x \neq \theta
$$

take place for some $\epsilon>0$ and $k<1 / 2$. Then for every $\delta, \tau>0$

$$
\lim _{t \rightarrow \infty} \mathcal{P}\left\{t^{1 / 2+\delta}|X(t)-\theta|<\tau \mid X(t) \rightarrow \theta\right\}=0 .
$$


Differentiability of the urn function at the limit point is a highly demanding restriction, as well as the fact that the urn function has to be independent of $t$. As said before, several actual markets can present overwhelming problems of formalization. Consequently, it may be impossible to check these conditions, albeit intuitively there is no reason why differentiability should matter. Conversely, we can obtain a general result in terms of $L^{2}$-convergence that suffices to the task:

Proposition 4 Let $\theta \in(0,1)$ be such that

$$
[f(t, x)-\theta](x-\theta)>k(x-\theta)^{2} \quad \text { for } \quad x \in(\theta-\epsilon, \theta+\epsilon), x \neq \theta
$$

takes place eventually for some $\epsilon>0$ and $k<1$. Then for every $\delta>0$

$$
\lim _{t \rightarrow \infty} t^{2 \min \{1-k, 1 / 2\}+\delta} \mathcal{P}\{X(t) \rightarrow \theta\} \int_{\{X(t) \rightarrow \theta\}}(X(t)-\theta)^{2} d \mathcal{P} \neq 0 .
$$

The proposition is proved in the appendix.

Proposition 2 and 4 show that convergence to 0 and 1 can be much faster (almost of order $1 / t$ as $t \rightarrow \infty$ ) than to an interior limit (which can be almost of order $1 / \sqrt{t}$ only $)^{1112}$. Here $t$ stands for the number of adoptions to the urn. That is, we are talking about relative rates (the ideal time which is considered here is the time of product selection choices). This result is however stronger than it may seem at first glance. In fact it has also implications for the patterns of product selection in "real" (empirical) time where plausibly the speed of the market share trajectory depends also on technology adoption decisions. There is much qualitative evidence and some econometric results [e.g. Koski and Nijkamp (1997)] showing that technology adoption is at the very least independent of market shares if not enhanced by increasing asymmetry in their distribution. Thus a fortiori we can conclude that there is a natural tendency of this class of processes to converge faster to 0 or 1 rather than to an interior limit. The explanation is that the variance of $\zeta^{t}(x)$, which characterizes the level of random disturbances in the process $(2)$, is $f(t, x)(1-f(t, x))$. Under condition (9) this value vanishes at 0 and 1 but it

\footnotetext{
${ }^{11}$ If returns are constant, the result of proposition 3 and 4 simply becomes the wellknown textbook result on rate of convergence of the sample mean and its variance.

${ }^{12}$ Bassanini and Dosi (1999b) shows that $2 \min \{1-k, 1 / 2\}$ is also an upper bound to the rate of convergence to an interior limit, therefore proposition 4 could be written in an even stronger way, although not necessary for the task of the present paper.
} 
does not vanish at $\theta \in(0,1)$, being equal to $\theta(1-\theta)$, under condition (10). Notice also that in example $1 c=0$ and in example $2 c \cong 0$.

As shown in the previous section, the urn function can have any shape and there is no reason to believe that problems characterized by 0 and 1 as the only stable points are the only ones that we can expect. Therefore, in principle, an asymptotic outcome where both technologies survive should be observable with positive frequency in real markets. As discussed in the previous section the tendency to converge to market sharing or technological monopolies is an outcome induced by the relative impact of heterogeneity of preferences and increasing returns to adoption. What tendency is realized depends on which of the two prevails. Notice however that the prevalence of one of the two factors is not always predictable ex ante even for a nearly omniscient agent fully aware of all fundamentals of the economy: in the examples of the previous section both type of outcomes are possible, but which one is realized depends on the actual sequence of historical events that lead to it. In this type of models, in general, when multiple asymptotic equilibria are attainable, history plays a major role in the selection of the actual one ${ }^{13}$.

If asymptotic patterns were observable, the results of the previous section would imply that we should observe both stable market sharing and technological monopolies. However for the interpretation of empirical stylized facts, the point where the process eventually would converge may be irrelevant. Indeed, the rate of change of the technological and economic environment can be sufficiently high that one can always observe diffusion dynamics well short of any meaningful neighborhood of the limit it would have attained under forever constant external conditions. So while it is true that a convergent process should generate a long-lasting stable pattern, the time required to generate it may be too long to actually observe it: the world is likely to change well before convergence is actually attained. In a sense these changes can be viewed as resetting the game to its starting point.

On the basis of the propositions of this section we notice that convergence to technological monopolies tends to be much faster (in probabilistic terms) than to any stable market sharing where both technologies coexist, because of the intrinsic variability that market sharing carries over. Thus the empirical prediction of these results can be stated as follows: in markets with increasing returns to adoption and a high rate of technological change we expect to

${ }^{13}$ For a general discussion on this point see also Dosi (1997). 
observe a prevalence of unstable market sharing (persistent fluctuations in the market shares) and stable technological quasi-monopolies over stable patterns of market sharing. The reason for this being that technological monopolies can be easily attained in a reasonably short time, i.e. sufficiently before any significative change in the underlying basic technological paradigms [Dosi (1982)].

Finally note that the observation of the frequent emergence of different monopolies in different related markets (e.g. different geographical areas) does not contradict our empirical predictions. Of course it is trivially true that, with mutually independent markets, different trajectories could emerge in different markets as if they were different realizations of the same experiment. In a related paper [Bassanini and Dosi (1999a)] we show that the foregoing results can be extended also to the case when markets are interdependent: Not contrary to the intuition, it is the balance between local and global feedbacks which determines whether the system converges to the same or different monopolies in every market. However, even though at high level of aggregation a system of different local monopolies looks like a stable market sharing, it is shown there that it has the same rate-of-convergence properties of a "univariate" system converging to a monopoly.

\section{Conclusions}

This paper has reassessed the empirical evidence on prevalence of technological monopolies over market-sharing in the dynamics of competing technologies. First we have argued that the dominant explanation in the literature, namely that unbounded increasing returns can be identified as the factor responsible for this pattern, does not always hold. Brian Arthur's results - we have shown - hold only when increasing returns to adoption are linear or more than linear and the degree of heterogeneity of agents is small. The presented counterexamples suggest that asymptotic patterns of the dynamics of competing technologies depend on the relative impact of (unbounded) increasing returns and the degree of heterogeneity of the population of adopters. Second, given all this, we propose however that in a market with high technological dynamism, no interesting predictions can be made by simply looking at theoretical asymptotic patterns: If convergence is too slow the environment changes before the limit can be actually approached. Conversely, developing upon some mathematical properties of Polya urns, we show that convergence 
to technological monopolies tends to be (in probabilistic terms) much faster than to a limit where both technologies coexist, the empirical implication being that in markets with high turnover of basic technologies, a prevalence of technological monopolies over stable market sharing is likely to be observed.

\section{Appendix}

For the purpose of exposition, to keep the notation simple, all proofs are exposed for generalized urn scheme involving time-independent urn functions. They can be easily repeated for the general case.

Proof of proposition 1. Consider only the first case - convergence to 0 . Without loss of generality we can assume that $\mathcal{P}\{X(t) \rightarrow 0\}>0$. Indeed the theorem, being a statement about the convergence rate to 0 , does not make any sense if $X(\cdot)$ does not converge to 0 .

Let $Z(\cdot)$ be a conventional urn process with $c x$ as the urn-function and the same initial numbers of balls $n=n^{w}+n^{b}$. Then

$$
E Z(t+1) \leq\left(1-\frac{1-c}{t+n}\right) E Z(t), \quad t \geq 1,
$$

and consequently

$$
\begin{aligned}
& E Z(t) \leq E Z(1) \prod_{j=1}^{t-1}\left(1-\frac{1-c}{j+n}\right) \leq E Z(1) e^{c-1 \sum_{j=1}^{t-1} \frac{1}{(n+j)}} \leq \\
& \leq E Z(1) e^{c-1} \int_{n+1}^{n+t-1} \frac{1}{x} d x=E Z(1)\left(\frac{n+1}{n+t-1}\right)^{1-c}=E Z(1) t^{c-1}\left[1+o_{t}(1)\right] \text {, }
\end{aligned}
$$

where $o_{t}(1) \rightarrow 0$ as $t \rightarrow \infty$. Hence from Chebychev's inequality

$$
\mathcal{P}\left\{t^{1-c-\delta} Z(t)>\tau\right\} \rightarrow 0 \text { as } t \rightarrow \infty
$$

for every $\delta \in(0,1-c)$ and $\tau>0$.

For arbitrary $\sigma \in(0, \epsilon)$ and $v>0$ there is $N$ depending on these variables such that

$$
\mathcal{P}\{\{X(t) \rightarrow 0\} \Delta\{X(s) \leq \sigma, s \geq N\}\}<v,
$$


where $A \Delta B=(A \backslash B) \cup(B \backslash A)$. Also since $Z(t) \rightarrow 0$ with probability 1 as $t \rightarrow \infty$, we can choose this $N$ so large that

$$
\mathcal{P}\{\{X(t) \rightarrow 0\} \Delta\{X(s) \leq \sigma, Z(s) \leq \sigma, s \geq N\}\}<v .
$$

To prove the theorem it is enough to show that

$$
\mathcal{P}\left\{t^{1-c-\delta} X(t)>\tau, X(t) \rightarrow 0\right\} \rightarrow 0,
$$

or, taking into account that $v$ in (12) can be arbitrary small, that

$$
\mathcal{P}\left\{t^{1-c-\delta} X(t)>\tau, X(s) \leq \sigma, Z(s) \leq \sigma, s \geq N\right\} \rightarrow 0
$$

However

$$
\begin{gathered}
\mathcal{P}\left\{t^{1-c-\delta} X(t)>\tau, X(s) \leq \sigma, Z(s) \leq \sigma, s \geq N\right\}= \\
=\sum_{y \in S_{N}^{X}} \mathcal{P}\left\{\begin{array}{l}
t^{1-c-\delta} X(t)>\tau, X(s) \leq \sigma, \quad \mid X(N)=y \\
Z(s) \leq \sigma, s \geq N \\
\mathcal{P}\{X(N)=y\},
\end{array}\right.
\end{gathered}
$$

where $S_{t}^{X}=\left\{\frac{X(1)(n+i)}{n+t-1}, 0 \leq i \leq t-1\right\}$ is the set of values that $X(t)$ can attain (not necessarily with positive probability). Due to lemma 2.2 of Hill et al. (1980), there exists a probability space such that $Z(\cdot)$ dominates $X(\cdot)$ on the event $Z(t) \leq \sigma, t \geq N$, providing that these processes start from the same point. Therefore, for any $y \in S_{N}^{X}$

$$
\begin{aligned}
& \mathcal{P}\left\{t^{1-c-\delta} X(t)>\tau, X(s) \leq \sigma, Z(s) \leq \sigma, s \geq N \mid X(N)=y\right\} \leq \\
& \leq \mathcal{P}\left\{t^{1-c-\delta} Z(t)>\tau, X(s) \leq \sigma, Z(s) \leq \sigma, s \geq N \mid X(N)=y\right\} .
\end{aligned}
$$

However for every $y \in S_{N}^{X}$

$$
\begin{aligned}
& \mathcal{P}\left\{t^{1-c-\delta} Z(t)>\tau, X(s) \leq \sigma, Z(s) \leq \sigma, s \geq N\right\} \leq \\
& \leq \mathcal{P}\left\{t^{1-c-\delta} Z(t)>\tau\right\} \rightarrow 0
\end{aligned}
$$

as $t \rightarrow \infty$ by (11). Thus (14) is a sum of a finite number - namely $N$ - of terms each converging to zero. This completes the proof. 
Proof of proposition 2. As before, consider only the first case - convergence to 0 .

Let $Z(\cdot)$ be a conventional urn process with $c x$ as the urn-function and the same initial numbers of balls $n=n^{w}+n^{b}$. Then

$$
\begin{aligned}
& E\left[Z(t+1)^{2} \mid Z(t)\right]=Z(t)^{2}+ \\
& +\frac{2}{n+t}(c-1) Z(t)^{2}+\frac{1}{(n+t)^{2}}(c-1)^{2} Z(t)^{2}+ \\
& +\frac{1}{(n+t)^{2}} c(1-c)^{2} Z(t)^{2} .
\end{aligned}
$$

and

$$
E\left[Z(t+1)^{2}\right]=\left(1-\frac{2(1-c)}{t+n}+\frac{1-c}{(t+n)^{2}}\right) E\left[Z(t)^{2}\right], \quad t \geq 1,
$$

and consequently

$$
\begin{aligned}
& E\left[Z(t)^{2}\right]=E\left[Z(1)^{2}\right] \prod_{j=1}^{t-1}\left[1-\frac{2(1-c)}{j+n}+o_{j}(1 / j)\right] \leq \\
& \leq E\left[Z(1)^{2}\right] e^{2(c-1) \sum_{j=1}^{t-1} \frac{1}{(n+j)}} \leq E\left[Z(1)^{2}\right] e^{2(c-1) \int_{n+1}^{n+t-1} \frac{1}{x} d x}= \\
& =E\left[Z(1)^{2}\right]\left(\frac{n+1}{n+t-1}\right)^{2(1-c)}=E\left[Z(1)^{2}\right] t^{2(c-1)}\left[1+o_{t}(1)\right] \text {, }
\end{aligned}
$$

where $t^{s} o_{t}\left(1 / t^{s}\right) \rightarrow 0$ as $t \rightarrow \infty$. Hence:

$$
t^{2(1-c-\delta)} E\left[Z(t)^{2}\right] \rightarrow 0 \text { as } t \rightarrow \infty
$$

for every $\delta \in(0,1-c)$.

For arbitrary $\sigma \in(0, \epsilon)$ and $v>0$ there is $N$ depending on these variables such that

$$
\mathcal{P}\{\{X(t) \rightarrow 0\} \Delta\{X(s) \leq \sigma, s \geq N\}\}<v,
$$

where $A \Delta B=(A \backslash B) \cup(B \backslash A)$. Also since $Z(t) \rightarrow 0$ with probability 1 as $t \rightarrow \infty$, we can choose this $N$ so large that

$$
\mathcal{P}\{\{X(t) \rightarrow 0\} \Delta\{X(s) \leq \sigma, Z(s) \leq \sigma, s \geq N\}\}<v .
$$

To prove the theorem it is enough to show that

$$
t^{2(1-c-\delta)} \int_{\{X(t) \rightarrow 0\}} X(t)^{2} d \mathcal{P} \rightarrow 0,
$$


or, taking into account that $v$ in (16) can be arbitrary small, that

$$
t^{2(1-c-\delta)} \int_{\{X(s) \leq \sigma, Z(s) \leq \sigma, s \geq N\}} X(t)^{2} d \mathcal{P} \rightarrow 0,
$$

However

$$
\begin{aligned}
& t^{2(1-c-\delta)} \int_{\{X(s) \leq \sigma, Z(s) \leq \sigma, s \geq N\}} X(t)^{2} d \mathcal{P}= \\
& =\sum_{y \in S_{N}^{X}} t^{2(1-c-\delta)} \int_{\{X(s) \leq \sigma, Z(s) \leq \sigma, s \geq N, X(N)=y\}} X(t)^{2} d \mathcal{P}
\end{aligned}
$$

where $S_{t}^{X}=\left\{\frac{X(1)(n+i)}{n+t-1}, 0 \leq i \leq t-1\right\}$ is the set of values that $X(t)$ can attain (not necessarily with positive probability). Due to lemma 2.2 of Hill et al. (1980), there exists a probability space such that $Z(\cdot)$ dominates $X(\cdot)$ on the event $Z(t) \leq \sigma, t \geq N$, providing that these processes start from the same point. Therefore, for any $t \geq N$ and $y \in S_{N}^{X}$

$$
\begin{aligned}
& \int_{\{X(s) \leq \sigma, Z(s) \leq \sigma, s \geq N, X(N)=y\}} X(t)^{2} d \mathcal{P} \leq \\
& \leq t^{2(1-c-\delta)} \int_{\{X(s) \leq \sigma, Z(s) \leq \sigma, s \geq N, X(N)=y\}} Z(t)^{2} d \mathcal{P} .
\end{aligned}
$$

However for every $y \in S_{N}^{X}$

$$
\begin{aligned}
& t_{\{X(s) \leq \sigma, Z(s) \leq \sigma, s \geq N, X(N)=y\}} Z(t)^{2} d \mathcal{P} \leq \\
& \leq t^{2(1-c-\delta)} E\left[Z(t)^{2}\right] \rightarrow 0
\end{aligned}
$$

as $t \rightarrow \infty$ by (15). Thus (18) is a sum of a finite number - namely $N$ - of terms each converging to zero. This completes the proof. 
Proof of proposition 4. The proof is based on the following lemmas ${ }^{14}$ :

Lemma 1 Let $f($.$) be the urn function of the process Z(t)$ such that

$$
[f(x)-\theta](x-\theta)>k(x-\theta)^{2}
$$

for some $k<1$ and $\theta \in(0,1)$ and $f(x)[1-f(x)] \geq \alpha>0$. Then $\lim _{t \rightarrow \infty} d_{t}=$ $\lim _{t \rightarrow \infty} E(Z(t)-\theta)^{2}$, where

$$
d_{t} \geq K\left\{\begin{array}{lll}
(n+t)^{-1} & \text { if } & 2(1-k)-1>0 \\
(n+t)^{-1} \log (n+t) & \text { if } & 2(1-k)-1=0 \\
(n+t)^{-2(1-k)} & \text { if } & 2(1-k)-1<0
\end{array}\right.
$$

where $K$ is a constant term.

Proof Consider the process (2) and write $n=n^{w}+n^{b}$, then:

$$
\begin{aligned}
& E\left[(Z(t+1)-\theta)^{2} \mid X(t)\right]=(Z(t)-\theta)^{2}+ \\
& +\frac{2}{n+t}[f(Z(t))-Z(t)](Z(t)-\theta)+\frac{1}{(n+t)^{2}}[f(Z(t))-Z(t)]^{2}+ \\
& +\frac{1}{(n+t)^{2}} f(Z(t))[1-f(Z(t))] .
\end{aligned}
$$

Setting $\Delta_{t}=E(Z(t)-\theta)^{2}$, from the assumptions of the lemma, taking into account that $f(Z(t))[1-f(Z(t))] \geq \alpha$, and that

$$
[f(Z(t))-Z(t)](Z(t)-\theta)=[f(Z(t))-\theta](Z(t)-\theta)-(Z(t)-\theta)^{2},
$$

we have

$$
\Delta_{t+1} \geq \Delta_{t}\left[1-\frac{2(1-k)}{n+t}\right]+\frac{\alpha}{(n+t)^{2}}
$$

Thus

$$
\Delta_{t+1} \geq \Delta_{1} \prod_{i=1}^{t}\left[1-\frac{2(1-k)}{n+i}\right]+\sum_{i=1}^{t} \frac{\alpha}{(n+i)^{2}} \prod_{j=i+1}^{t}\left[1-\frac{2(1-k)}{n+j}\right] .
$$

Since

$$
\prod_{j=i+1}^{t}\left[1-\frac{2(1-k)}{n+j}\right]=e^{-2(1-k)} \sum_{j=i+1}^{t} \frac{1}{(n+j)}\left(1+\beta_{i t}\right) \geq
$$

${ }^{14}$ We are indebted to Yuri Kaniovski for suggesting us the line of the following proof. 


$$
\geq e^{-2(1-k)[\log (n+t-1)-\log (n+i)]}\left(1+\beta_{i t}\right)=\left(\frac{n+i}{n+t}\right)^{2(1-k)}\left(1+\gamma_{i t}\right),
$$

where $\beta_{i t}$ and $\gamma_{i t}$ are small terms $\left(o_{t}(1)\right)$ not necessarily non-negative ${ }^{15}$, then

$$
\begin{aligned}
\Delta_{t+1} \geq & \Delta_{1}\left(\frac{n+1}{n+t}\right)^{2(1-k)}\left(1+\gamma_{1 t}\right)+ \\
& +\alpha(n+t)^{-2(1-k)} \sum_{i=1}^{t}(n+i)^{-2+2(1-k)}\left(1+\gamma_{i t}\right) .
\end{aligned}
$$

Since, in terms of asymptotic behavior,

$$
\sum_{i=1}^{t}(n+i)^{-2+2(1-k)} \cong\left\{\begin{array}{lll}
\frac{1}{2(1-k)-1}(n+t)^{2(1-k)-1} & \text { if } 2(1-k)-1 \neq 0 \\
\log (n+t) & \text { if } 2(1-k)-1=0
\end{array}\right.
$$

we have that

$$
\Delta_{t+1} \geq K\left\{\begin{array}{lll}
\frac{1}{2(1-k)-1}(n+t)^{-1} & \text { if } & 2(1-k)-1>0 \\
(n+t)^{-1} \log (n+t) & \text { if } & 2(1-k)-1=0 \\
\Delta_{1}(n+t)^{-2(1-k)} & \text { if } & 2(1-k)-1<0
\end{array}\right.
$$

which implies the statement of the lemma.

Set

$$
\begin{aligned}
& \tilde{f}(x)=\left\{\begin{array}{lll}
f(\theta-\epsilon) & \text { if } \quad x<\theta-\epsilon \\
f(x) & \text { if } \theta-\epsilon<x<\theta+\epsilon, \\
f(\theta+\epsilon) & \text { if } x>\theta+\epsilon
\end{array}\right. \\
& \tilde{X}(t+1)=\tilde{X}(t)+\frac{\tilde{\xi}^{t}(\tilde{X}(t))-\tilde{X}(t)}{t+n}, \\
& \tilde{X}(1)=X(1) .
\end{aligned}
$$

Then, with probability $1, \tilde{X}(t) \rightarrow \theta$ as $t \rightarrow \infty$. Also by lemma 1 ,

$$
t^{2 \min \{1-k, 1 / 2\}+\delta} E\left[(\tilde{X}(t)-\theta)^{2}\right] \nrightarrow 0 \text { as } t \rightarrow \infty .
$$

${ }^{15}$ The line of reasoning here is the same as for the proof of proposition 1 and 2. 
As for proposition 2, we can ignore the case when $X(t)$ does not converge with positive probability.

We have to show that as $t \rightarrow \infty$

$$
t^{2 \min \{1-k, 1 / 2\}+\delta} \int_{\{X(t) \rightarrow \theta\}}(X(t)-\theta)^{2} d \mathcal{P} \nrightarrow 0,
$$

For every $\sigma>0$ there is a $t(\sigma)$ such that

$$
\mathcal{P}\{\{X(s) \rightarrow \theta\} \Delta\{|X(t)-\theta|<\epsilon, t \geq t(\sigma)\}\} \leq \sigma .
$$

Since $\sigma$ can be arbitrarily small, (20) holds if and only if

$$
t^{2 \min \{1-k, 1 / 2\}+\delta} \int_{\{|X(s)-\theta|<\epsilon, s \geq t(\sigma)\}}(X(t)-\theta)^{2} d \mathcal{P} \nrightarrow 0 .
$$

However

$$
\begin{aligned}
& t^{2 \min \{1-k, 1 / 2\}+\delta} \int_{\{|X(s)-\theta|<\epsilon, s \geq t(\sigma)\}}(X(t)-\theta)^{2} d \mathcal{P}= \\
& =t^{2 \min \{1-k, 1 / 2\}+\delta} \sum_{y \in S_{t(\sigma)}} \int_{\{|X(s)-\theta|<\epsilon, s \geq t(\sigma), X(t(\sigma))=y\}}(X(t)-\theta)^{2} d \mathcal{P},
\end{aligned}
$$

where $S_{t}=\tilde{S}_{t}=\left\{\frac{X(1)(n+i)}{n+t-1}, 0 \leq i \leq t-1\right\}$ is the set of values that $X(t)$ and $\tilde{X}(t)$ can attain (not necessarily with positive probability). Notice that for any $t>t(\sigma)$ and $y \in S_{t(\sigma)}$

$$
\begin{aligned}
& \int_{\{|X(s)-\theta|<\epsilon, s \geq t(\sigma), X(t(\sigma))=y\}}(X(t)-\theta)^{2} d \mathcal{P}= \\
& =\int_{\{|\tilde{X}(s)-\theta|<\epsilon, s \geq t(\sigma), \tilde{X}(t(\sigma))=y\}}(\tilde{X}(t)-\theta)^{2} d \mathcal{P} .
\end{aligned}
$$

This follows from the fact that $\tilde{f}(x)$ and $f(x)$ are the same for $x \in[\theta-\epsilon, \theta+\epsilon]$. However

$$
t^{2 \min \{1-k, 1 / 2\}+\delta} \sum_{y \in S_{t(\sigma)}} \int_{\{|\tilde{X}(s)-\theta|<\epsilon, s \geq t(\sigma), \tilde{X}(t(\sigma))=y\}}(\tilde{X}(t)-\theta)^{2} d \mathcal{P}=
$$




$$
\begin{aligned}
& =t^{2 \min \{1-k, 1 / 2\}+\delta} \int_{\{\tilde{X}(t) \rightarrow \theta\}}(\tilde{X}(t)-\theta)^{2} d \mathcal{P}= \\
& =t^{2 \min \{1-k, 1 / 2\}+\delta} E\left[(\tilde{X}(t)-\theta)^{2}\right] \nrightarrow 0,
\end{aligned}
$$

as $t \rightarrow \infty$ by (19). This completes the proof.

\section{References}

Arthur, W.B., On Competing Technologies and Historical Small Events: The Dynamics of Choice under Increasing Returns, IIASA Working Paper WP-83-90 [Reprinted in W.B. Arthur (1994) Increasing Returns and PathDependence in the Economy, Ann Arbor: University of Michigan Press], 1983.

Arthur, W.B., Self Reinforcing Mechanisms in Economics, in P.W. Anderson, K.Arrow and R.Pines (eds.), The Economy as an Evolving Complex System, New York: Addison-Wesley, 1988.

Arthur, W.B., Competing Technologies, Increasing Returns and Lock-In by Historical Events, Econ. J., 99, 116-131, 1989.

Arthur, W.B., "Sylicon Valley" Locational Clusters: When Do Increasing Returns Imply Monopoly?, Math. Soc. Sci., 19, 235-51, 1990.

Arthur, W.B., Y.Ermoliev and Y.Kaniovski, Generalized Urn Problem and Its Applications, Cybernetics, 19, 61-71, 1983.

Arthur, W.B., Y.Ermoliev and Y.Kaniovski, Strong Laws for a Class of Path-Dependent Urn Processes, Proceedings of the International Conference on Stochastic Optimization, Lecture Notes on Control and Information Sciences, 81, 187-300, 1986.

Arthur, W.B. and D.Lane, Information Contagion, Structural Change and Econ. Dynamics, 4, 81-104, 1993.

Banerjee, A., A Simple Model of Herd Behavior, Quart. J. Econ., 107, 797-817, 1992.

Bassanini, A.P., Localized Technological Change and Path-Dependent Growth, IIASA Interim Report IR-97-086, 1997.

Bassanini, A.P. and G.Dosi, Heterogeneous Agents, Complementarities, and Diffusion of Technologies: Do Increasing Returns Imply Convergence to International Monopolies?, in D.Delli Gatti, M.Gallegati and A.Kirman 
(eds.), Market Structure, Aggregation and Heterogeneity, Berlin: Springer, forthcoming, 1999a.

Bassanini, A.P. and G.Dosi, Competing Technologies, Technological Monopolies and the Rate of Convergence to a Stable Market Structure, Laboratory of Economics and Management Working Paper n.3, Sant 'Anna School of Advanced Studies, 1999b.

Bernheim, B.D., A Theory of Conformity, J. Pol. Econ., 102, 841-77, 1994.

Breuhan, A.L., Innovation and the Persistence of Technological Lock-In, unpublished manuscript, 1996.

Brock, W.A. and S.N.Durlauf, Discrete Choice with Social Interactions I: Theory, NBER Working Paper \#5291, 1995.

Brynjolfsson, E. and C.F.Kemerer, Network Externalities in Microcomputer Software: An Econometric Analysis of the Spreadsheet Market, Management Science, 42, 1627-47, 1996.

Church, J. and N.Gandal, Complementary Network Externalities and Technological Adoption, Int. J. of Industrial Organization, 11, 239-60, 1993.

Cowan, R., Nuclear Power Reactors: A Study in Technological Lock-In, J. of Econ. History, 50, 541-67, 1990.

Cowan, R., Tortoises and Hares: Choice among Technologies of Unknown Merit, Econ. J., 101, 801-14, 1991.

Cowan, R., and W.Cowan, Technological Standardization with and without Borders in an Interacting Agents Model, unpublished paper, 1998.

Cusumano, M.A., Y.Milonadis and R.S.Rosenbloom, Strategic Maneuvering and Mass-Market Dynamics: The Triumph of VHS over Beta, Business History Rev., 66, 51-94, 1992.

David, P., Clio and the Economics of QWERTY, AEA Papers and Proceedings, 75, 332-7, 1985.

David, P., Heroes, Herds and Hystheres in Technological Theory: Thomas Edison and the Battle of Systems Reconsidered, Industrial and Corporate Change, 1, 129-80, 1992.

Dosi, G., Technological Paradigms and Technological Trajectories, Research Policy, 11, 142-67, 1982.

Dosi, G., Opportunities, Incentives and the Collective Patterns of Technological Change, Econ. J., 107, 1530-47, 1997.

Dosi, G., Y.Ermoliev and Y.Kaniovski, Generalized Urn Schemes and Technological Dynamics, J. Math. Econ., 23, 1-19, 1994. 
Economides, N., The Economics of Networks, Int. J. of Industrial Organization, 14, 673-99, 1996.

Farrell, J. and G.Saloner, Standardization, Compatibility and Innovation, Rand J. of Econ., 16, 70-83, 1985.

Farrell, J. and G.Saloner, Installed Base and Compatibility: Innovation, Product Preannouncements, and Predation, Amer. Econ. Rev., 76, 940-55, 1986.

Hill, B.M., D.Lane and W.Sudderth, A Strong Law for Some Generalized Urn Processes, Annals of Probability, 8, 214-26, 1980.

Islas, J., Getting Round the Lock-In in Electricity Generating Systems: The Example of the Gas Turbine, Research Policy, 26, 49-66, 1997.

Katz, M.L. and C.Shapiro, Network Externalities, Competition , and Compatibility, Amer. Econ. Rev., 75, 424-40, 1985.

Katz, M.L. and C.Shapiro, Technology Adoption in the Presence of Network Externalities, J. Pol. Econ., 94, 822-41, 1986.

Katz, M.L. and C.Shapiro, Product Introduction with Network Externalities, J. of Industrial Econ., 40, 55-84, 1992.

Katz, M.L. and C.Shapiro, Systems Competition and Network Effects, J. of Econ. Perspectives, 8, 93-115, 1994.

Koski, H. and P.Nijkamp, The Installed Base Effect: Some Empirical Evidence from the Microcomputer Market, unpublished manuscript, 1997.

Krugman, P., History vs. Expectations, Quart. J. Econ., 106, 651-67, 1991a.

Krugman, P., Geography and Trade, Cambridge, MA: MIT press, 1991b.

Liebowitz, S.J. and S.E.Margolis, Network Externality: An Uncommon Tragedy, J. of Econ. Perspectives, 8, 133-150, 1994.

Oren, S. and S.Smith, Critical Mass and Tariff Structure in Electronic Communications Markets, Bell J. of Econ., 12, 467-87, 1981.

Rauch, J.E., Does History Matter Only When It Matters Little? The Case of City-Industry Location, Quart. J. Econ., 108, 843-67, 1993.

Rohlfs, J., A Theory of Interdependent Demand for a Communication Service, Bell J. of Econ., 5, 16-37, 1974.

Rosenberg, N., Inside the Black Box, Cambridge: Cambridge University Press, 1982.

Saviotti, P.P. and A.Trickett, The Evolution of Helicopter Technology, 1940-1986, Economics of Innovation and New Technology, 2, 111-30, 1992.

Tushman, M.L. and J.P.Murmann, Dominant Designs, Technology Cycles, and Organizational Outcomes, in B.Staw and L.L.Cummings (eds.), 
Research in Organizational Behavior, 20, Greenwich, CT: JAI press, forthcoming, 1998.

Venables, A.J., Localization of Industry and Trade Performance, Oxford Rev. of Econ. Policy, 12(3), 52-60, 1996.

Walker, S. and P.Muliere, Beta-Stacy Processes and a Generalisation of the Polya-urn Scheme, Annals of Statistics, 25, 1762-80, 1997.

Winter, S.G., Comments on Arrow and Lucas, J. of Business, 59, S42734, 1986. 